\title{
Effect of self-phase-modulation on dispersion compensated absolute polar duty cycle division multiplexing transmission
}

\begin{abstract}
The effect of self-phase modulation (SPM) on $40 \mathrm{~Gb} / \mathrm{s}$ absolute polar duty cycle division multiplexing (AP-DCDM) is investigated and reported. The study includes the influence of launched power, number of channels and dispersion compensation method. Dispersion postcompensation and combination of dispersion pre- and post-compensation are used to manage the transmission links. At high powers, SPM degrades the pulse recompression process and provides an upper bound on the AP-DCDM transmitted pulse energy. It is demonstrated that the $40 \mathrm{~Gb} / \mathrm{s}$ AP-DCDM system shows a $4.1 \mathrm{~dB}$ improvement and less than $1 \mathrm{~dB}$ penalty in terms of SPM tolerance in comparison to $40 \mathrm{~Gb} / \mathrm{s} 4$-ary and on off-keying (OOK) systems, respectively. The SPM effect is stronger in the 100 post-compensated link than that in the combination of pre- and post-compensated links. Dispersion pre-compensation of 1822 is found as the optimum range of pre-compensation ratio for AP-DCDM system, which makes optimisation of the launched power possible.
\end{abstract}

